\title{
Bardet-Biedl Syndrome: A Rare Report of two Sibling Affected and Third Sibling with Multiple Anomalies
}

\author{
MD. FIROZANJUM ${ }^{1}$, MD. AZIZUR RAHMAN¹, RANJIT RANJAN ROY²
}

\section{Introduction}

BBS is named after Georges Bardet and Arthur Biedl. The first known case was reported by Laurence and Moon in 1866. Laurence-Moon-Biedl-Bardet syndrome (LMBBS) is no longer considered as a valid term as patients of Laurence and Moon had paraplegia but no polydactyly and obesity, which are the key elements of the BBS. Hence, LaurenceMoon syndrome is usually considered as a separate entity. However, some recent research suggests that the two conditions may not be distinct. Bardet-Biedl syndrome (BBS) is a rare ciliopathic autosomal recessive disorder. ${ }^{1}$ The prevalence of BBS varies markedly between populations from 1:160000 in northern European to $1: 13500$ and 1:175000 respectively in isolated communities in Kuwait and Newfoundland, where a higher level of consanguinity prevails. ${ }^{2,3}$ BBS is part of a group of human genetic disorders of cilia function, and mutations of 17 genes are reported to be responsible for more than $80 \%$ of clinically diagnosed cases. ${ }^{4}$ Cilia are cellular structures of two classes: motile and immotile. Motile cilia generate movement of fluid. Defects of motile cilia can be manifested in the form of bronchiectasis and infertility. On the other hand, immotile cilia function as sensory organelles and their defects are characterized clinically by retinitis pigmentosa, polydactyly, situs inversus, learning difficulties and cystic formation in kidneys, liver and pancreas. BBS is a disorder of immotile cilia. ${ }^{2}$

Major features of the syndrome are rod-cone dystrophy (generally causing blindness), polydactyly, central obesity, renal abnormalities and genital anomalies (hypogenitalism in men) and learning difficulties. Minor features are speech delay, development delay,

1. MD Resident, Phase B, Department of Pediatric Nephrology, Bangabandhu Sheikh Mujib Medical University, Shahbag, Dhaka.

2. Professor, Department of Pediatric Nephrology, Bangabandhu sheikh Mujib Medical University, Shahbag, Dhaka.

Correspondence: Dr. Md Firoz Anjum, MD Resident, Phase B, Department of Pediatric Nephrology, Bangabandhu Sheikh Mujib Medical University, Shahbag, Dhaka, Email: firoz.anjum2013@gmail.com. diabetes mellitus, dental anomalies, congenital heart disease, brachydactyly/syndactyly, ataxia/poor coordiantion, facial dysmorphism, anosmia/hyposmia, hypertension. The presence of four major features or three major plus two minor features establishes the clinical diagnosis of the syndrome. Among these manifestations, only post-axial polydactyly is apparent at birth. Obesity appears frequently during childhood, learning difficulties du-ring school age and visual difficulties by the teen years. ${ }^{5}$

There is widespread controversy whether Bardet Biedl syndrome and Laurence -Moon syndrome are separate entity or whether they are simply phenotypic variation of the same disorder. Our interest is to report three cases of multiple anomalies, two of them was diagnosed BBS and one case having multiple anomalies. All three children are from same parents and suffering from a rare disease.

\section{Case reports}

Two out of three cases fulfilling the clinical diagnostic criteria for BBS (Table 1) and one case having multiple anomalies. All cases were diagnosed at the Department of Pediatric Nephrology, Bangabandhu Sheikh Mujib Medical University (BSMMU), Dhaka.

\section{Case-1}

A 10 year old boy, $2^{\text {nd }}$ issue of his $1^{\text {st }}$ degree consanguineous parents, visited in pediatric nephrology outpatient department (OPD) with the complaints of excessive weight gain, very short length of penis and absent left sided testis. He has no history of dribbling of urine, frequency $f$ urine, hematuria, trauma to urethra, dental problem, jaundice and cardiac surgery during early life. He was born by normal vaginal delivary without complication. He has developmental delay evidenced by started to walk by 2 years of his age and started to speak 2-3 word sentences by 3 years of age. On examination he was well alert, not pallor, jaundice absent, oral cavity normal, BCG mark was present, lymph node not enlarged, post axial polydactyly of right lower limbs is present, spine normal. Temperature $99.6^{\circ} \mathrm{F}$, Pulse rate 100 beats 
per minutes, respiratory rate 20 breaths per minutes, blood pressure $105 / 60 \mathrm{mmHg}$ (on $50^{\text {th }}$ centile), height $140 \mathrm{~cm}\left(50^{\text {th }}\right.$ centile), weight $50 \mathrm{~kg}$ ( $97^{\text {th }}$ centile), body mass index (BMI) of $25 \mathrm{~kg} / \mathrm{m}^{2}$ (BMI for age on $97^{\text {th }}$ centile), intelligence quotient (IQ) score is 80 . Ear, nose and throat reveals normal finding. Genitourinary examination system micropenis present (stretched penile length is $50 \mathrm{~mm},-3.63 \mathrm{SD}$ ) (figure 1) with absent left testis (figure 2), kindey and urinary bladder not palpable and renal angle non tender. Nervous system and cardiovascular examination: reveals normal finding. Eye examination: astigmatism and nystagmus present in left eye, visiual acuity was $6 / 36$ and normal retina on fundoscopy. Laboratory examination including complete blood count, urinalysis, ultrasonography of whole abdomen, renal function tests, thyroid function tests were found to be normal. Blood glucose $6 \mathrm{mmol} / \mathrm{l}$. HbA1c was not done as his blood glucose was within normal range. Liver biopsy was not done as there was no features of liver disease.

\section{Case- 2}

The most younger daughter of the same parents, she was five years and six months old. She is suffering from incontinence of urine and visual disturbance since birth. She had anorectal malformation which was corrected at 21 days of her age. She also had post axial polydactyly in right hand and left toe which was corrected at the same time. She has no history of

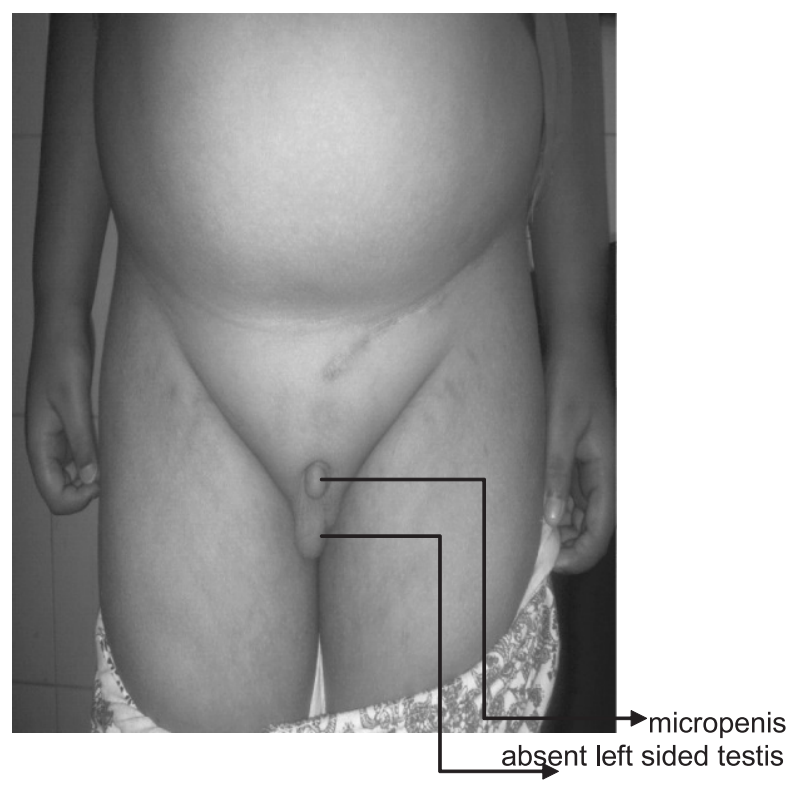

Fig.-1

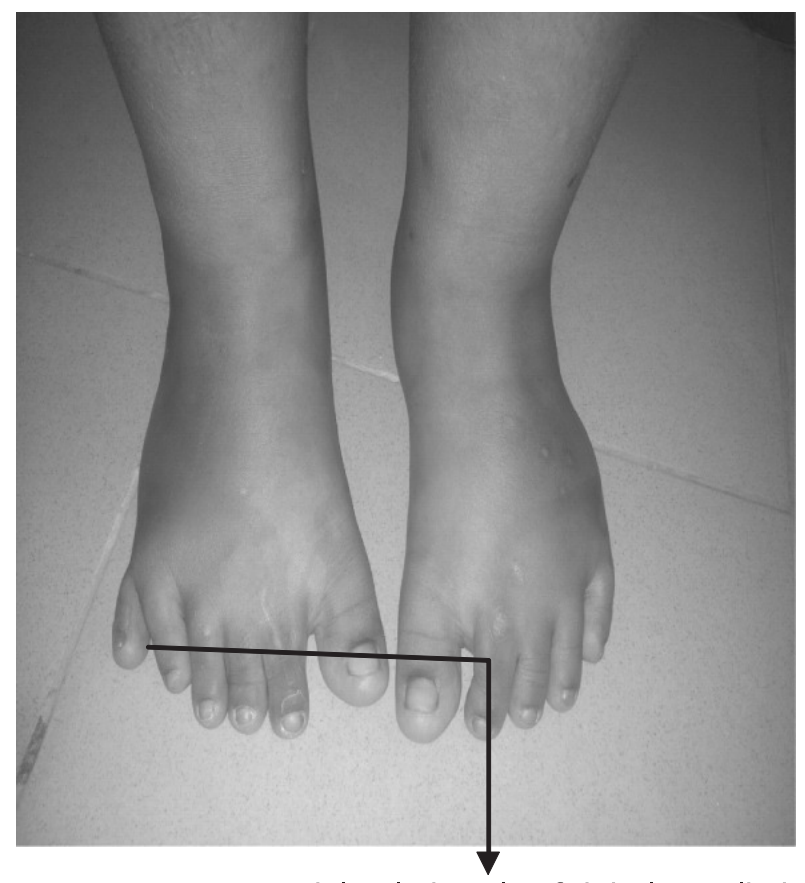

post axial polydactyly of right lower limb

Fig.-2

fever, hematuria, dental problem and jaundice and cardiac surgery during early life. She was born by normal vaginal delivary without complication. She is developmentally age appropriate. On examination she was well alert, not pallor, jaundice absent, oral cavity normal, BCG mark was present, lymph node not enlarged, scar mark present over little finger of right hand and left toe, spine reveals lumber lordosis. Temperature $99.6^{\circ} \mathrm{F}$, pulse rate 110 beats per minutes, respiratory rate 22 breaths per minutes, blood pressure $105 / 68 \mathrm{mmHg}$ (on $90^{\text {th }}$ centile), Height $102 \mathrm{~cm}\left(10^{\text {th }}\right.$ centile), Weight $27 \mathrm{~kg}$ ( $97^{\text {th }}$ centile), body mass index (BMI) of $27 \mathrm{~kg} / \mathrm{m}^{2}$ (BMI for age ( $97^{\text {th }}$ centile), intelligence quotient (IQ) score is 85 . Ear, nose and throat reveals normal finding. Genitourinary system, nervous system and cardiovascular examination: reveals normal finding. Eye examination: strabismus present in left eye, visiual acuity was $6 / 12$ in both eye and normal retina on fundoscopy. Laboratory examination including complete blood count, urinalysis, renal function tests, spot urinary calcium creatinine ratio and spot urinary uric acid creatinine ratio, thyroid function tests were found to be normal. Blood glucose $7 \mathrm{mmol} / \mathrm{l}$. HbA1c was not done as her blood glucose was within normal range. X-ray of 
lumbosacral spine revealed lumbar lordosis. Ultrasonography of kidney-ureter -bladder (USG of KUB) revealed multiple calcified areas at apices of pyramid of both kidneys. Liver biopsy was not done as there was no features of liver disease (Figure 3).

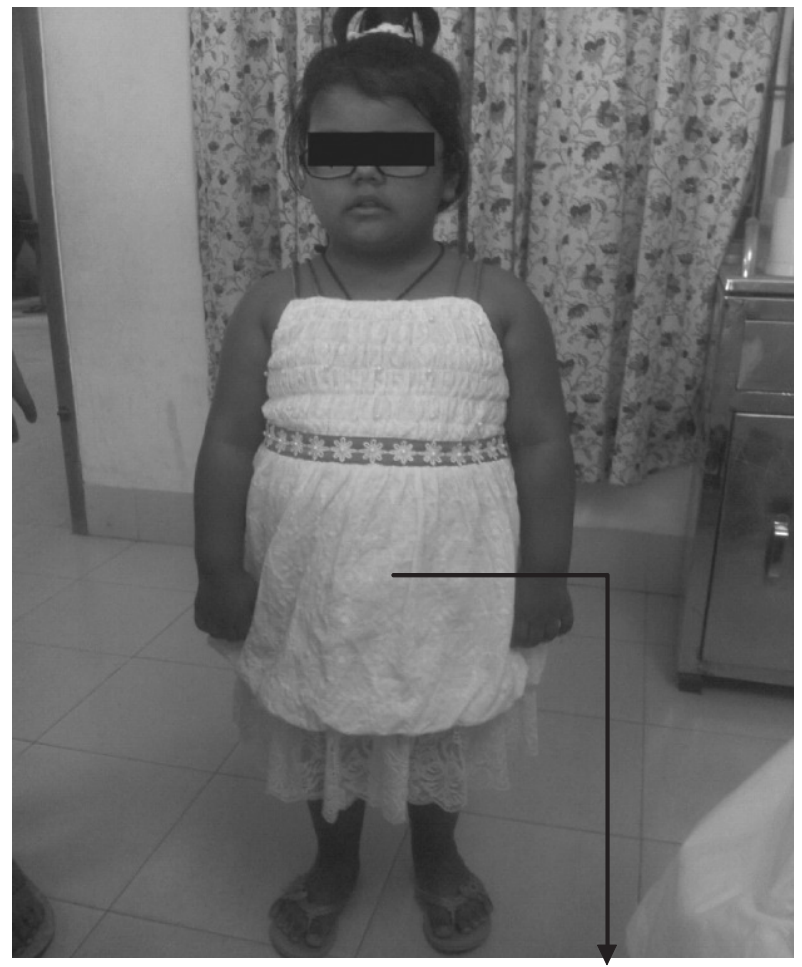

obese child

\section{Fig.-3}

\section{Case-3}

A 12 year old girl, $1^{\text {st }}$ issue of same parents. She is suffering from night time bed wetting since birth, weakness of the lower limbs and difficulty in sitting from standing. She was never dry since birth. She had no history of fever, hematuria, dental problem and jaundice, trauma or fall from height or cardiac surgery during early life. She was born by normal vaginal delivary without complication. She is developmentally age appropriate. On examination she was well alert, not pallor, jaundice absent, oral cavity normal, BCG mark was present, lymph node not enlarged, spine reveals swelling over lumber region. Temperature $99.6^{0}$ F, Pulse rate 100 beats per minutes, Respiratory rate 16 breaths per minutes, Blood pressure $110 / 75 \mathrm{mmHg}$ (between $50^{\text {th }}-90^{\text {th }}$ centile), Height $144 \mathrm{~cm}$ (between $10^{\text {th }}-25^{\text {th }}$ centile), Weight $45 \mathrm{~kg}\left(50^{\text {th }}-75^{\text {th }}\right.$ centile $)$, body mass index (BMI) of $21 \mathrm{~kg} / \mathrm{m}^{2}$ (BMI for age between $50^{\text {th }}-75^{\text {th }}$ centile), intelligence quotient (IQ) score is 85 . Ear, nose and throat reveals normal finding. Genitourinary system, nervous system and cardiovascular examination: reveals normal finding. Eye examination: reveals normal finding. Lower limb muscle power was mildly diminished (grade $3 / 4$ ), gait is ataxic. All the deep tendon reflexes of lower limbs were normal. Laboratory examination including complete blood count, urinalysis, renal function tests, serum electrolyte, serum creatine phosphokinase, serum aldolase, spot urinary calcium creatinine ratio, serum osmolality and urine osmolality were found to be normal. Blood glucose $5 \mathrm{mmol} / \mathrm{l}$. HbA1c was not done as her blood glucose was within normal range. $X$-ray of lumbosacral spine revealed spondylolithesis of L4 near L5 and spondylolysis of L5 vertebra. USG of KUB region detect no abnormality but post voidal residual volume $30 \mathrm{ml}$. MRI of brain was normal but electroencephalogram (EEG) revealed repeated bouts of high voltage sharp and slow waves in both hemispheres consistent with seizure disorder. Liver biopsy was not done as there was no features of liver disease. She was diagnosed as a case of primary nocturnal enuresis with seizure disorder (Figure 4).

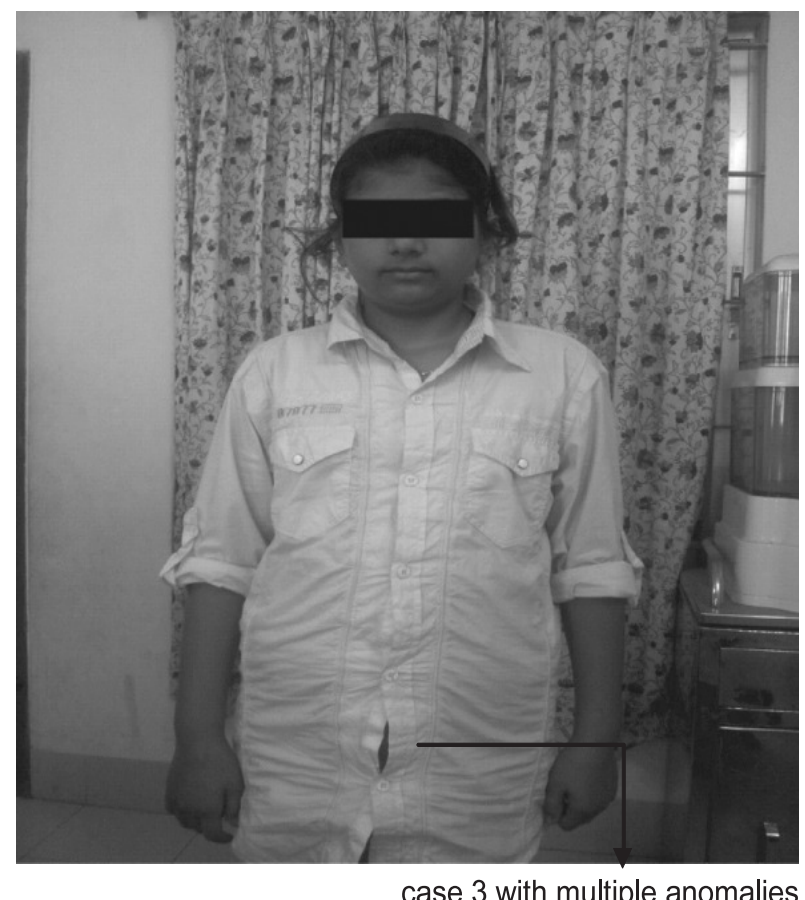

Fig. -4 
Table-I

Diagnostic features and prevalence in BBS

\begin{tabular}{|c|c|c|c|c|}
\hline & Frequency & Case-1 & Case-2 & Case-3 \\
\hline \multicolumn{5}{|l|}{ Primary features } \\
\hline Rod-cone dystrophy & $93 \%$ & - & - & - \\
\hline Polydactyly & $63-81 \%$ & + & + & - \\
\hline Obesity & $72-92 \%$ & + & + & - \\
\hline Genital anomalies & $59-98 \%$ & + & - & - \\
\hline Renal anomalies & $53 \%$ & - & + & + \\
\hline Learning difficulties & $61 \%$ & - & - & - \\
\hline \multicolumn{5}{|l|}{ Secondary features } \\
\hline Speech delay & $54-81 \%$ & + & - & - \\
\hline Developmental delay & $50-91 \%$ & + & - & - \\
\hline Diabetes mellitus & $6-48 \%$ & - & - & - \\
\hline Dental anomalies & $51 \%$ & - & - & - \\
\hline Congenital heart disease & $7 \%$ & - & - & - \\
\hline Brachydactyly/ syndactyly & $6-100 \% / 8-95 \%$ & - & - & - \\
\hline Ataxia/ poor coordination & $40-86 \%$ & - & - & + \\
\hline Mild spasticity & & - & - & - \\
\hline Anosmia/hyposmia & $60 \%$ & - & - & - \\
\hline \multicolumn{2}{|c|}{ Strabismus/cataracts/astigmatism } & + & + & - \\
\hline \multicolumn{2}{|c|}{ Hepatic fibrosis } & - & - & - \\
\hline \multicolumn{2}{|l|}{ Spinal problem } & - & + & + \\
\hline
\end{tabular}

Four primary features or three primary features and two secondary features are required for a clinical diagnosis of Bardet-Biedl syndrome. ${ }^{5}$

\section{Discussion}

Bardet-Biedl Syndrome(BBS) is rare autosomal recessive ciliopathy characterized by retinal dystrophy, obesity, post-axial polydectyly, renal dysfunction, learning difficulties and hypogonadism. ${ }^{1}$ Apart from the cardinal manifestations, other features of the BBS include various degrees of intellectual impairment, congenital heart block, brachycephaly, deafness and dental anomalies. The full spectrum of clinical features is found in only $40-45 \%$ of BBS cases. ${ }^{6}$ Hypogonadism, which is probably primary in origin, is reported more frequently in males than in females. ${ }^{7}$

The diagnosis of Bardet-Biedl syndrome (BBS) is established by clinical findings. Beales et al. have suggested that the presence of four primary features or three primary features plus two secondary features are diagnostic (Table-1). ${ }^{5}$ Case 1 had three primary features and three secondary features, case 2 had three primary features and two secondary features but case 3 had only one primary feature and two secondary feature. So, case 1 and 2 fulfilling the diagnostic criteria for BBS but case 3 do not fulfilling the BBS criteria although having multiple anomalies.

Retinal dystrophy (100\%) is the first major feature of the disorder. It is found occasionally in the first decade but present in almost all patients by the second decade. ${ }^{8}$ Case 1 had astigmatism and nystagmus, case 2 had only visual disturbance but all three cases were having normal retina.

Obesity is the second major feature of BBS, with a frequency of 72-92 percent. Obesity usually begins in childhood and the severity increases with age, with the majority of cases exhibiting symptoms within the first year of life..$^{5}$ In this report, case 1 and case 2 is 
having obesity but case 3 had normal weight. Limbabnormalities are the third major feature of BBS. Limb deformities have been reported at varying frequencies. Of these, post-axial polydactyly and brachydactyly of both hands and feet are more common. Partial syndactyly, fifth finger clinodactyly, and a prominent gap between the first and second toes are sometimes associated. ${ }^{5}$ Case 1 had post axial polydactyly of right lower limbs, case 2 also had post axial polydactyly in right hand and left toe but case 3 had no any limbs deformities. Mental retardation is a more disputed feature of BBS. Recently, objective IQ tests determined that only a minority of patients are mentally retarded. An IQ of 79 or below is found in 44 per cent of BBS patients. The decrease in IQ level correlates with the presence of visual handicap. ${ }^{5}$ In this report all three cases had normal IQ. Hypogenitalism is reportedly more frequent in BBS males than females. ${ }^{5}$ In BBS females, genital abnormalities encompass a wide range, including hypoplastic fallopian tubes, uterus, and ovaries, partial and complete vaginal atresia, absent vaginal orifice, and absent urethral orifice. ${ }^{9,10}$ Bardet-Biedl syndrome males have small penis and testes (88\%). ${ }^{11}$ In these three cases, only case 1 had micropenis and left sided absent testis and other two cases had normal gonads and genitalia.

Renal failure is the major cause of morbidity and early mortality in BBS. A wide range of renal abnormalities has been described including chronic renal failure, parenchymal cysts, calyceal clubbing, fetal lobulation, scarring, unilateral agenesis, dysplastic kidneys, renal calculi, vesico-ureterix reflux. ${ }^{5}$ case 1 had normal renal function, case 2 had incontinence of urine since birth and USG of KUB shows multiple calcified areas at apices of pyramid of both kidney, case 3 had night time bed wetting since birth and USG of KUB region detect no abnormality but post voidal residual volume was $30 \mathrm{ml}$.

BBS is an autosomal recessive disorder characterized by nonallelic heterogeneity. BBS is genetically, heterogeneous with mutations of 17 genes are reported to be responsible for more than $80 \%$ of clinically diagnosed cases. ${ }^{4}$ In these cases genetic studies was not done due to inadequate facility.

Although currently there is no definitive treatment for BBS, early diagnosis is important to guide the management of the child through regular assessment of blood pressure, weight, renal imaging studies and function, ophthalmologic examinations and psychological support. Even neonatal diagnosis is possible by combining prenatal detection of polydactyly with ultrasonography plus genetic studies shortly after birth. ${ }^{12}$ Gynecologists and pediatricians have more chance to make an early diagnosis of the syndrome. Nevertheless, nephrologists have a main role in the treatment of the syndrome, since renal failure is the most frequent cause of death among BBS patients. ${ }^{13}$ In particular, pediatric nephrologists should be prepared to suspect the syndrome when treating children with renal tract abnormalities.

\section{Conclusion}

Though a lot of progress has been made about this rare disease, there are still more things need to be known about its pathophysiology. Further studies are required to understand the genetic complexity of Bardet-Biedl Syndrome. The disease is incurable, and therefore, persists as a chronic condition. However, timely symptomatic treatment ensures a good prognosis.

\section{References}

1. Moore SJ, Green JS, Fan Y, Bhogal AK, Dicks $\mathrm{E}$, Fernandez BA, et al. Clinical and genetic epidemiology of Bardet-Biedl syndrome in Newfoundland: A22-year prospective, population based, cohort study. Am J Med Genet. 2005;132:352-56.

2. Forsythe E, Beales PL. Bardet-Biedl Syndrome. Eur J Hum Genet. 2013; 21: 8-13.

3. Farag TI, TeebiAS. High incidence of Bardet Biedl syndrome among the Bedouin. Clin Genet. 1989;36:463-64.

4. Rodriguez MC, Irigoín F, Osborn DP, Gascue C, Katsanis N, Beales PL, et al. The Bardet-Biedl syndrome-related protein CCDC28B modulates mTORC2 function and interacts with SIN1 to control cilia length independently of the mTOR complex. Hum Mol Genet. 2013;22:4031-42.

5. Beales PL, Elcioglu N, Woolf AS, Parker D, Flinter FA. New criteria for improved diagnosis of Bardet-Biedl syndrome: re-sults of a population survey. J Med Genet. 1999; 36:43746.

6. Prosperi L, Cordella M, Bernasconi S. Electroretinography and diagnosis of the Laurence-Moon-Bardet-Biedl syndrome in 
childhood. J Pediatr Ophthalmol. 1977;14: 305-08.

7. Green JS, Parfrey PS, Harnett JD, Farid NR, Cramer BC, Johnson G, et al. The cardinal manifestations of Bardet-Biedl syndrome, a form of Laurence-Moon-Biedl syndrome. N Engl J Med. 1989;321:1002-09.

8. Croft JB, Morrell D, Chase CL, Swift M. Obesity in heterozygous carriers of the gene for the Bardet- Biedl syndrome. Am J Med Genet. 1995;55:12-15.

9. Mehrotra N, Taub S, Covert RF. Hydrometrocolpos as a neonatal manifestation of the Bardet-Biedl syndrome. Am J Med Genet. 1997;69:220.
10. Stoler JM, Herrin JT, Holmes LB. Genital abnormalities in females with Bardet-Biedl syndrome. Am J Med Genet. 1995;55:276-78.

11. Jones KL. Bardet-Biedl syndrome: Smith's Recognizable Patterns of Human Malformation 5th edition, saunders company. 1997;590-91.

12. Ashkinadze E, Rosen T, Brooks SS, Katsanis $N$, Davis EE. Combining fetal sonography with genetic and allele pathogenicity studies to secure a neonatal diagnosis of Bardet-Biedl syndrome. Clin Genet. 2013;83:553-59.

13. O'Dea D, Parfrey PS, Harnett JD, Hefferton D, Cramer BC, Green J. The importance of renal impairment in the natural history of Bardet-Biedl Syndrome. Am J Kidney Dis. 1996;27:776-83. 